\title{
Effects of Slip and Hall on the Peristaltic Transport of a Hyperbolic Tangent Fluid in a Planar Channel
}

\author{
M. Anusha Bai ${ }^{1}$ \\ ${ }^{1}$ Research Scholar, Department of Mathematics, \\ Sri Krishnadevaraya University, Ananthapuramu-515003, \\ A.P., India
}

\begin{abstract}
The effects of slip and Hall on the peristaltic transport of a hyperbolic tangent fluid in a planar channel under the assumption of long wavelength. The expressions for the velocity and axial pressure gradient are obtained by employing perturbation technique. The effects of Weissenberg number, power-law index, Hall parameter, Hartmann number and amplitude ratio on the axial pressure gradient and time-averaged volume flow rate are studied with the aid of graphs.
\end{abstract}

Keywords: Peristaltic Transport, Hyperbolic Tangent fluid, Planar Channel and Slip and Hall parameter

\section{INTRODUCTION}

Some fluids which are encountered in chemical applications do not adhere to the classical Newtonian viscosity prescription and are accordingly known as non-Newtonian fluids. One especial class of fluids which are of considerable practical importance is that in which the viscosity depends on the shear stress or on the flow rate. The viscosity of most nonNewtonian fluids, such as polymers, is usually a nonlinear decreasing function of the generalized shear rate. This is known as shear-thinning behavior. Such fluid is a hyperbolic tangent fluid (Ai and Vafai, 2005). Nadeem and Akram (2009) have first investigated the peristaltic flow of a hyperbolic tangent fluid in an asymmetric channel. Nadeem and Akbar (2011) have analyzed the peristaltic transport of a Tangent hyperbolic fluid in an endoscope numerically.

Based on Experimental controls, it was shown that the controlled application of low intensity and frequency pulsing magnetic fields could modify cell and tissue behavior. Biochemistry has taught us that cells are formed of positive or negative charged molecules. This is why these magnetic fields applied to living organisms may induce deep modifications in molecule orientation and in their interaction. An impulse magnetic field in the combined therapy of patients with stone fragments in the upper urinary tract was experimentally studied by Li et al. (1994). It was found that impulse magnetic

\author{
Prof. R. Sivaprasad ${ }^{2}$ \\ ${ }^{2}$ Professor, Department of Mathematics, \\ Sri Krishnadevaraya University, Ananthapuramu-515003, \\ A.P., India
}

field (IMF) activates impulse activity of ureteral smooth muscles in $100 \%$ of cases. Elshahed and Haroun (2005) have investigated the peristaltic flow of a Johnson-Segalman fluid in a planar channel under the effect of a magnetic field. Hayat and Ali (2006) have investigated the peristaltic motion of a MHD third grade fluid in a tube. Hayat et al. (2007) have first investigated the Hall effects on the peristaltic flow of a Maxwell fluid trough a porous medium in channel. Magnetohydrodynamic peristaltic flow of a hyperbolic tangent fluid in a vertical asymmetric channel with heat transfer was studied by Nadeem and Akram (2011). Eldabe (2015) have studied the Hall Effect on peristaltic flow of third order fluid in a porous medium with heat and mass transfer. Hall effects on the peristaltic pumping of a hyperbolic tangent fluid in a planar channel were studied by Subba Narasimhudu and Subba Reddy (2017).

The peristaltic flow of a Newtonian fluid through a two-dimensional micro channel with the slip effect was first investigated by Kwang (2000). El Sehaway et al. (2006) have studied the effect of slip on the peristaltic motion of a Maxwell fluid in a two-dimensional channel. The effects of slip and nonNewtonian parameters on the peristaltic transport of a third grade fluid in a circular cylindrical tube were investigated by Ali et al. (2009). Chaube et al. (2010) have analyzed the slip effects on the peristaltic flow of a micropolar fluid in a channel. Effects of slip and induced magnetic field on the peristaltic flow of pseudoplastic fluid were analyzed by Noreen et al. (2011). Subba Reddy et al. (2012) have investigated the slip effects on the peristaltic motion of a Jeffrey fluid through a porous medium in an asymmetric channel under the effect of magnetic field. Akbar et al. (2012) have discussed the peristaltic flow of a hyperbolic tangent fluid in an inclined asymmetric channel with slip and heat transfer. Slip effects on peristaltic transport of a Prandtl fluid in a channel under the effect of magnetic field was studied by Jyothi et al. (2015). 


\section{MATHEMATICAL FORMULATION}

We consider the peristaltic motion of a hyperbolic tangent fluid in a two-dimensional symmetric channel of width $2 a$ under the effect of magnetic field. The flow is generated by sinusoidal wave trains propagating with constant speed $c$ along the channel walls. A uniform magnetic field $B_{0}$ is applied in the transverse direction to the flow. The magnetic Reynolds number is considered small and so induces magnetic field neglected. Fig. 1 represents the physical model of the channel.

The wall deformation is given by

$Y= \pm H(X, t)= \pm a \pm b \cos \frac{2 \pi}{\lambda}(X-c t)$,

where $b$ is the amplitude of the wave, $\lambda$ - the wave length and $X$ and $Y$ - the rectangular co-ordinates with $X$ measured along the axis of the channel and $Y$ perpendicular to $X$. Let $(U, V)$ be the velocity components in fixed frame of reference $(X, Y)$.

The flow is unsteady in the laboratory frame $(X, Y)$

- However, in a co-ordinate system moving with the propagation velocity c (wave frame $(x, y)$ ), the boundary shape is stationary. The transformation from fixed frame to wave frame is given by

$$
x=X-c t, y=Y, u=U-c, v=V
$$

where $(u, v)$ and $(U, V)$ are velocity components in the wave and laboratory frames respectively.

The constitutive equation for a Hyperbolic Tangent fluid is

$\tau=-\left[\eta_{\infty}+\left(\eta_{0}+\eta_{\infty}\right) \tanh (\Gamma \dot{\gamma})^{n}\right] \dot{\gamma}$

where $\tau$ is the extra stress tensor, $\eta_{\infty}$ is the infinite shear rate viscosity, $\eta_{o}$ is the zero shear rate viscosity, $\Gamma$ is the time constant, $n$ is the power-law index and $\dot{\gamma}$ is defined as

$$
\dot{\gamma}=\sqrt{\frac{1}{2} \sum_{i} \sum_{j} \dot{\gamma}_{i j} \dot{\gamma}_{j i}}=\sqrt{\frac{1}{2} \pi}
$$

where $\pi$ is the second invariant stress tensor. We consider in the constitutive equation ( 3 ) the case for which $\eta_{\infty}=0$ and $\Gamma \dot{\gamma}<1$, so the Eq. (3) can be written as

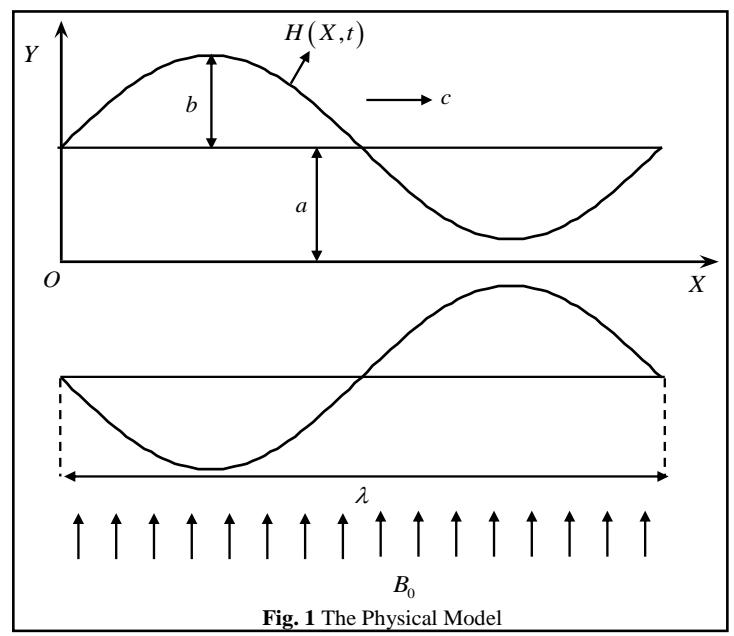

$\tau=-\eta_{0}(\Gamma \dot{\gamma})^{n} \dot{\gamma}=-\eta_{0}(1+\Gamma \dot{\gamma}-1)^{n} \dot{\gamma}=-\eta_{0}(1+n[\Gamma \dot{\gamma}-1]) \dot{\gamma}$

The above model reduces to Newtonian for $\Gamma=0$ and $n=0$.

The equations governing the flow in the wave frame of reference are

$$
\frac{\partial u}{\partial x}+\frac{\partial v}{\partial y}=0
$$

$\rho\left(u \frac{\partial u}{\partial x}+v \frac{\partial u}{\partial y}\right)=-\frac{\partial p}{\partial x}-\frac{\partial \tau_{x x}}{\partial x}-\frac{\partial \tau_{y x}}{\partial y}+\frac{\sigma B_{0}^{2}}{1+m^{2}}(m v-(u+c))$

$\rho\left(u \frac{\partial v}{\partial x}+v \frac{\partial u}{\partial y}\right)=-\frac{\partial p}{\partial y}-\frac{\partial \tau_{x y}}{\partial x}-\frac{\partial \tau_{y y}}{\partial y}-\frac{\sigma B_{0}^{2}}{1+m^{2}}(m(u+c)+v)$

where $\rho$ is the density $\sigma$ is the electrical conductivity, $B_{0}$ is the magnetic field strength and $m$ is the Hall parameter.

The corresponding dimensional boundary conditions are $u+\beta \tau_{x y}=-c \quad$ at $\quad y=H$ (slip condition) 


$$
\begin{aligned}
& \frac{\partial u}{\partial y}=0 \quad \text { at } \quad y=0 \\
& \text { (symmetry condition) }
\end{aligned}
$$

here $\beta$ is the slip parameter.

$$
\frac{\partial p}{\partial x}=\frac{\partial}{\partial y}\left\{\left[1+n\left(W e \frac{\partial u}{\partial y}-1\right)\right] \frac{\partial u}{\partial y}\right\}-\frac{M^{2}}{1+m^{2}}(u+1)
$$

Introducing the non-dimensional variables defined by

$$
\begin{aligned}
& \bar{x}=\frac{x}{\lambda}, \bar{y}=\frac{y}{a}, \bar{u}=\frac{u}{c}, \bar{v}=\frac{v}{c \delta}, \delta=\frac{a}{\lambda}, \bar{p}=\frac{p a^{2}}{\eta_{0} c \lambda}, \phi=\frac{b}{a} \\
& h=\frac{H}{a}, \bar{t}=\frac{c t}{\lambda}, \bar{\tau}_{x x}=\frac{\lambda}{\eta_{0} c} \tau_{x x}, \bar{\tau}_{x y}=\frac{a}{\eta_{0} c} \tau_{x y}, \bar{\tau}_{y y}=\frac{\lambda}{\eta_{0} c} \tau_{y y}, \\
& \operatorname{Re}=\frac{\rho a c}{\eta_{0}}, W e=\frac{\Gamma c}{a}, \bar{\gamma}=\frac{\dot{\gamma} a}{c}, \bar{q}=\frac{q}{a c}
\end{aligned}
$$

into the Equations (6) - (8), reduce to (after dropping the bars)

$$
\frac{\partial u}{\partial x}+\frac{\partial v}{\partial y}=0
$$

$\operatorname{Re} \delta\left(u \frac{\partial u}{\partial x}+v \frac{\partial u}{\partial y}\right)=-\frac{\partial p}{\partial x}-\delta^{2} \frac{\partial \tau_{x x}}{\partial x}-\frac{\partial \tau_{x y}}{\partial y}+\frac{M^{2}}{1+m^{2}}(m \delta v-(u+1))$

$\operatorname{Re} \delta^{3}\left(u \frac{\partial v}{\partial x}+v \frac{\partial v}{\partial y}\right)=-\frac{\partial p}{\partial y}-\delta^{2} \frac{\partial \tau_{x y}}{\partial y}-\delta \frac{\partial \tau_{y y}}{\partial y}-\frac{\delta M^{2}}{1+m^{2}}(m(u+1)+\delta v)$

where $\tau_{x x}=-2[1+n(W e \dot{\gamma}-1)] \frac{\partial u}{\partial x}$

$$
\begin{aligned}
& \tau_{x y}=-[1+n(W e \dot{\gamma}-1)]\left(\frac{\partial u}{\partial y}+\delta^{2} \frac{\partial v}{\partial x}\right) \\
& \tau_{y y}=-2 \delta[1+n(W e \dot{\gamma}-1)] \frac{\partial v}{\partial y}
\end{aligned}
$$$$
\dot{\gamma}=\left[2 \delta^{2}\left(\frac{\partial u}{\partial x}\right)^{2}+\left(\frac{\partial u}{\partial y}+\delta^{2} \frac{\partial v}{\partial x}\right)^{2}+2 \delta^{2}\left(\frac{\partial v}{\partial y}\right)^{2}\right]^{\frac{1}{2}}
$$
and

$M=a B_{0} \sqrt{\frac{\sigma}{\eta_{0}}}$ is the Hartmann number.

Under lubrication approach, neglecting the terms of order $\delta$ and Re, the Eqs. (13) and (14) become

$$
\frac{\partial p}{\partial y}=0
$$

From Eq. (15) and (16), we get

$$
\frac{d p}{d x}=(1-n) \frac{\partial^{2} u}{\partial y^{2}}+n W e \frac{\partial}{\partial y}\left[\left(\frac{\partial u}{\partial y}\right)^{2}\right]-\frac{M^{2}}{1+m^{2}}(u+1)
$$

The corresponding non-dimensional boundary conditions in the wave frame are given by

$$
\begin{aligned}
& u+\beta\left[1+n\left(W e \frac{\partial u}{\partial y}-1\right)\right] \frac{\partial u}{\partial y}=-1 \quad \text { at } \\
& y=h=1+\phi \cos 2 \pi x \\
& \frac{\partial u}{\partial y}=0 \quad \text { at } \quad y=0
\end{aligned}
$$

The volume flow rate $q$ in a wave frame of reference is given by

$$
q=\int_{0}^{h} u d y .
$$

The instantaneous flow $Q(X, t)$ in the laboratory frame is

$$
Q(X, t)=\int_{0}^{h} U d Y=\int_{0}^{h}(u+1) d y=q+h
$$

The time averaged volume flow rate $\bar{Q}$ over one period $T\left(=\frac{\lambda}{c}\right)$ of the peristaltic wave is given by

$$
\bar{Q}=\frac{1}{T} \int_{0}^{T} Q d t=q+1
$$




\section{SOLUTION}

Since Eq. (17) is a non-linear differential equation, it is not possible to obtain closed form solution. Therefore, we employ regular perturbation to find the solution.

For perturbation solution, we expand $u, \frac{d p}{d x}$ and $q$ as follows

$$
\begin{aligned}
& u=u_{0}+W e u_{1}+O\left(W e^{2}\right) \\
& \frac{d p}{d x}=\frac{d p_{0}}{d x}+W e \frac{d p_{1}}{d x}+O\left(W e^{2}\right) \\
& q=q_{0}+W e q_{1}+O\left(W e^{2}\right)
\end{aligned}
$$

Substituting these equations into the Eqs. (17) - (19), we obtain

\section{A. SYSTEM OF ORDER $W e^{0}$}

$\frac{d p_{0}}{d x}=(1-n) \frac{\partial^{2} u_{0}}{\partial y^{2}}-\frac{M^{2}}{1+m^{2}}\left(u_{0}+1\right)$

and the respective boundary conditions are

$$
\begin{aligned}
& u_{0}+\beta(1-n) \frac{\partial u_{0}}{\partial y}=-1 \quad \text { at } \quad y=h \\
& \frac{\partial u_{0}}{\partial y}=0 \quad \text { at } \quad y=0
\end{aligned}
$$

\section{B. SYSTEM OF ORDER $W e^{1}$}

$\frac{d p_{1}}{d x}=(1-n) \frac{\partial^{2} u_{1}}{\partial y^{2}}+\frac{\partial}{\partial y}\left[\left(\frac{\partial u_{o}}{\partial y}\right)^{2}\right]-\frac{M^{2}}{1+m^{2}} u_{1}$

and the respective boundary conditions are

$u_{1}+\beta(1-n) \frac{\partial u_{1}}{\partial y}+\beta n\left(\frac{\partial u_{0}}{\partial y}\right)^{2}=0$ at $\quad y=h$

$$
\frac{\partial u_{1}}{\partial y}=0 \quad \text { at } \quad y=0
$$

\section{SOLUTION FOR SYSTEM OF ORDER $W e^{0}$}

Solving Eq. (26) using the boundary conditions (27) and (28), we obtain

$$
u_{0}=\frac{1}{N^{2}(1-n)} \frac{d p_{0}}{d x}\left[\frac{\cosh N y}{a_{1}}-1\right]-1
$$

where $N=M / \sqrt{(1-n)\left(1+m^{2}\right)} \quad$ and

$a_{1}=\cosh N h+N \beta(1-n) \sinh N h$.

The volume flow rate $q_{0}$ is given by

$q_{0}=\frac{1}{N^{3}(1-n)} \frac{d p_{0}}{d x}\left[\frac{\sinh N h-N h a_{1}}{a_{1}}\right]-h$

From Eq. (A), we have

$$
\frac{d p_{0}}{d x}=\frac{\left(q_{0}+h\right) N^{3}(1-n) a_{1}}{\left[\sinh N h-N h a_{1}\right]}
$$

\section{SOLUTION FOR SYSTEM OF ORDER $W e^{1}$}

Substituting Eq. (32) in the Eq. (29) and solving the Eq. (29), using the boundary conditions (30) and (31), we obtain

$u_{1}=\frac{1}{N^{2}(1-n)} \frac{d p_{1}}{d x}\left[\frac{\cosh N y}{a_{1}}-1\right]+\frac{n}{3} \frac{\left(\frac{d p_{0}}{d x}\right)^{2}}{\left[N(1-n) a_{1}\right]^{3}} f(y)$

Where $f(y)=\left\{\begin{array}{l}{[(\sinh 2 N h-2 \sinh N h) \cosh N y+(2 \sinh N y-\sinh 2 N y) \cosh N h} \\ +N \beta(1-n)\left[\begin{array}{l}2(\cosh 2 N h-\cosh N h) \cosh N y-\sinh ^{2} N h \cosh N y \\ +\sinh N h(2 \sinh N y-\sinh 2 N y)\end{array}\right]\end{array}\right\}$ The volume flow rate $q_{1}$ is given by

$$
q_{1}=\frac{1}{N^{3}(1-n)} \frac{d p_{1}}{d x}\left[\frac{\sinh N h-N h a_{1}}{a_{1}}\right]+a_{2}\left(\frac{d p_{0}}{d x}\right)^{2}
$$


where

$a_{2}=n\left(\frac{(4-3 \cosh N h+2 \sinh 2 N h \sinh N h-\cosh 2 N h \cosh N h)+a_{3}}{6 N^{4}(1-n)^{3} a_{1}^{3}}\right)$

and

$a_{3}=N \beta(1-n)\left(3 \sinh N h(\cosh 2 N h-1)-2 \sinh ^{3} N h\right)$.

From Eq. (36) and (34), we have

$$
\frac{d p_{1}}{d x}=\frac{q_{1} N^{3}(1-n) a_{1}}{\left[\sinh N h-N h a_{1}\right]}-a_{4}\left(\frac{d p_{0}}{d x}\right)^{2}
$$

where

$a_{4}=n\left(\frac{4-3 \cosh N h+2 \sinh 2 N h \sinh N h-\cosh 2 N h \cosh N h+a_{3}}{6 N(1-n)^{2} a_{1}^{2}\left(\sinh N h-N h a_{1}\right)}\right)$

Substituting Equations (16) and (37) into the Eq. (24) and using the relation $\frac{d p_{0}}{d x}=\frac{d p}{d x}-W e \frac{d p_{1}}{d x}$ and neglecting terms greater than $O(W e)$, we get

$\frac{d p}{d x}=\frac{(q+h) N^{3}(1-n) a_{1}}{\left[\sinh N h-N h a_{1}\right]}-W e a_{4}\left(\frac{(q+h) N^{3}(1-n) a_{1}}{\left[\sinh N h-N h a_{1}\right]}\right)^{2}$

The dimensionless pressure rise per one wavelength in the wave frame is defined as

$$
\Delta p=\int_{0}^{1} \frac{d p}{d x} d x
$$

Note that, as $\beta \rightarrow 0$ our results coincide with the results of Subba Narasimhudu and Subba reddy (2017); as $\beta \rightarrow 0, m \rightarrow 0, M \rightarrow 0, W e \rightarrow 0$ and $n \rightarrow 0$ our results coincide with the results of Shapiro et al. (1969).

\section{RESULTS AND DISCUSSION}

In this section, we have carried out numerical calculations and plotted graphs to study effects of the Weissenberg number $W_{e}$, the power-law index $n$, the slip parameter $\beta$, the Hall parameter $m$, the Hartmann number $M$ and the/8- amplitude ratio $\phi$ on the axial pressure gradient and pumping characteristics.
Fig. 2 shows the variation of the axial pressure gradient $\frac{d p}{d x}$ with $W e$ for $n=0.5, m=0.2, \beta=0.1$, $M=1, \phi=0.5$ and $\bar{Q}=-1$. It is observed that, the axial pressure gradient $\frac{d p}{d x}$ increases with increasing Wiessenberg number $W e$.

The variation of the axial pressure gradient $\frac{d p}{d x}$ with $n$ for $W e=0.01, \quad m=0.2, \quad M=1, \quad \beta=0.1$, $\phi=0.5$ and $\bar{Q}=-1$ is depicted in Fig. 3. It is found that, the axial pressure gradient $\frac{d p}{d x}$ decreases with an increase in power-law index $n$.

Fig. 4 illustrates the variation of the axial pressure gradient $\frac{d p}{d x}$ with $\beta$ for $n=0.5, W e=0.01, M=1$, $m=0.2, \phi=0.5$ and $\bar{Q}=-1$. It is noted that, the axial pressure gradient $\frac{d p}{d x}$ decreases with increasing slip parameter $\beta$.

The variation of the axial pressure gradient $\frac{d p}{d x}$ with $m$ for $n=0.5, \quad W e=0.01, \quad M=1, \quad \beta=0.1$, $\phi=0.5$ and $\bar{Q}=-1$ shown in Fig. 5. It is noted that, the axial pressure gradient $\frac{d p}{d x}$ decreases with increasing Hall parameter $m$

Fig. 6 depicts the variation of the axial pressure gradient $\frac{d p}{d x} \quad$ with $M \quad$ for $n=0.5, \quad m=0.2$, $W e=0.01, \beta=0.1, \phi=0.5$ and $\bar{Q}=-1$. It is observed that, on increasing Hartmann number $M$ increases the axial pressure gradient $\frac{d p}{d x}$.

The variation of the axial pressure gradient $\frac{d p}{d x}$ with $\phi \quad$ for $\quad n=0.5, \quad m=0.2, \quad \beta=0.1, \quad M=1$, $W e=0.01$ and $\bar{Q}=-1$ is depicted in Fig. 7. It is found 
that, the axial pressure gradient $\frac{d p}{d x}$ increases with increasing amplitude ratio $\phi$.

Fig. 8 illustrates the variation of the pressure rise $\Delta p$ with $\bar{Q}$ for different values of We with $n=0.5$, $m=0.2, \beta=0.1, M=1$ and $\phi=0.5$. It is noted that, the time-averaged volume flow rate $\bar{Q}$ increases with increasing Wiessenberg number $W e$ in pumping $(\Delta p>0)$ , free-pumping $(\Delta p=0)$ and co-pumping $(\Delta p<0)$ regions.

The variation of the pressure rise $\Delta p$ with $\bar{Q}$ for different values of $n$ with $W e=0.01, \quad m=0.2$, $\beta=0.1, M=1$ and $\phi=0.5$ is shown in Fig. 9. It is found that, the time-averaged flow rate $\bar{Q}$ decreases with increasing $n$ in both the pumping and free pumping regions, while it increases with increasing $n$ in the co-pumping region.

Fig. 10 shows the variation of the pressure rise $\Delta p$ with $\bar{Q}$ for different values of $\beta$ with $n=0.5$, $W e=0.01, \beta=0.1, M=1$ and $\phi=0.5$. It is observed that, the time-averaged flow rate $\bar{Q}$ decreases with increasing $\beta$ in both the pumping region and the free pumping region, while it increases with increasing $\beta$ in the co-pumping region.

The variation of the pressure rise $\Delta p$ with $\bar{Q}$ for different values of $m$ with $n=0.5, W e=0.01$, $\beta=0.1, M=1$ and $\phi=0.5$ is illustrated in Fig. 11. It is observed that, the time-averaged flow rate $\bar{Q}$ decreases with increasing $m$ in the pumping region, while it increases with increasing $m$ in both the free pumping and co-pumping regions.

Fig. 12 depicts the variation of the pressure rise $\Delta p$ with $\bar{Q}$ for different values of $M$ with $n=0.5, \beta=0.1$ , $m=0.2, W e=0.01$ and $\phi=0.5$. It is noticed that, the time-averaged flow rate $\bar{Q}$ increases with increasing $M$ in the pumping region, while it decreases with increasing $M$ in both the free-pumping and co-pumping regions.
The variation of the pressure rise $\Delta p$ with $\bar{Q}$ for different values of $\phi$ with $n=0.5, m=0.2, \beta=0.1, M=1$ and $W e=0.01$ is depicted in Fig. 13. It is observed that, the time-averaged flow rate $\bar{Q}$ increases with increasing $\phi$ in both the pumping and free pumping regions, while it decreases with increasing $n$ in the co-pumping region for chosen $\Delta p(<0)$.
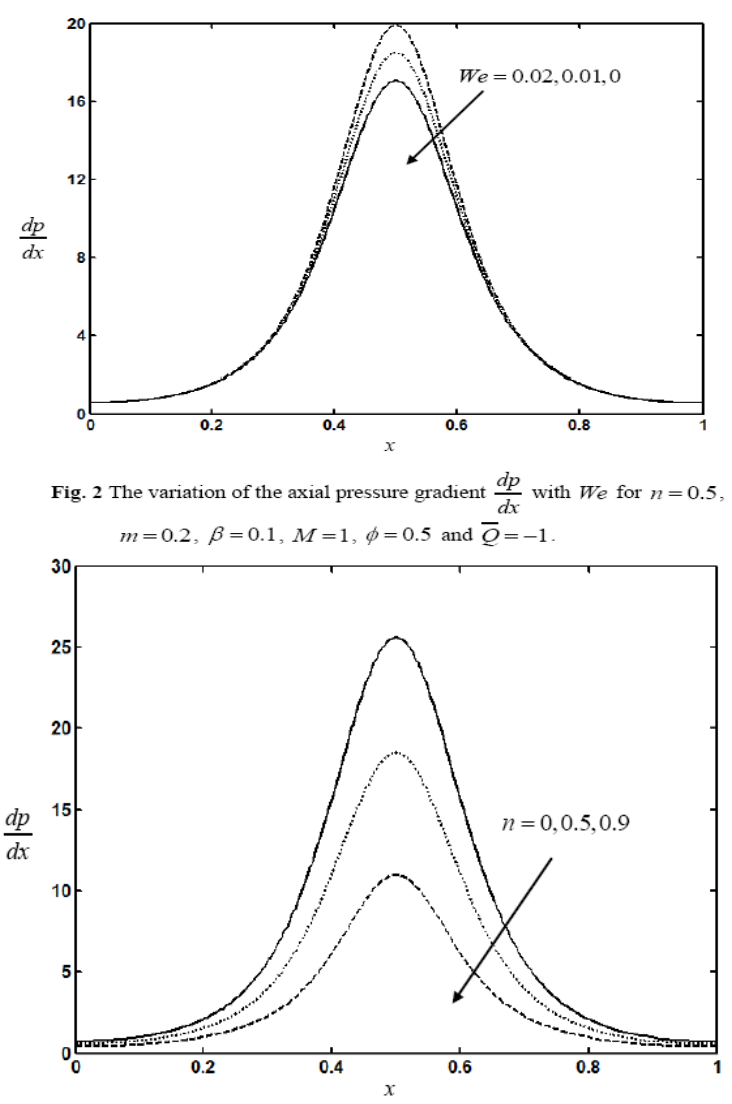

Fig. 3 The variation of the axial pressure gradient $\frac{d p}{d x}$ with $n$ for $W e=0.01$, $m=0.2, M=1, \beta=0.1, \phi=0.5$ and $\bar{Q}=-1$.

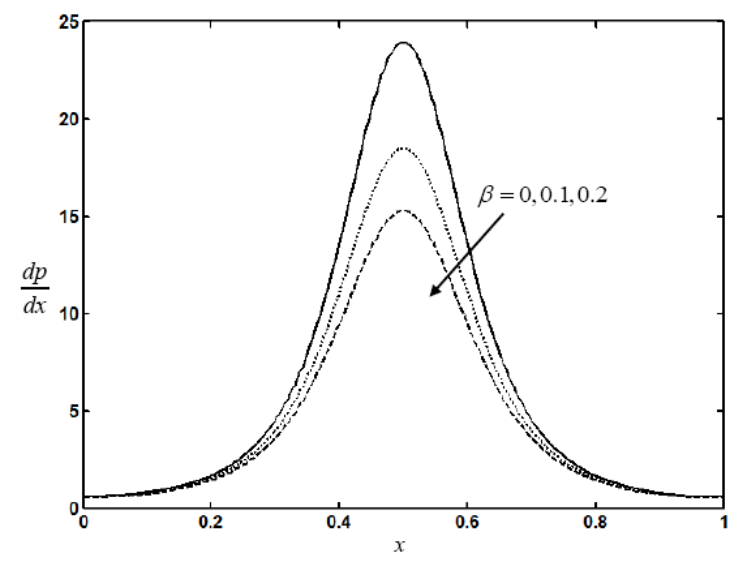

Fig. 4 The variation of the axial pressure gradient $\frac{d p}{d x}$ with $\beta$ for $n=0.5$, $W e=0.01, M=1, m=0.2, \phi=0.5$ and $\bar{Q}=-1$. 


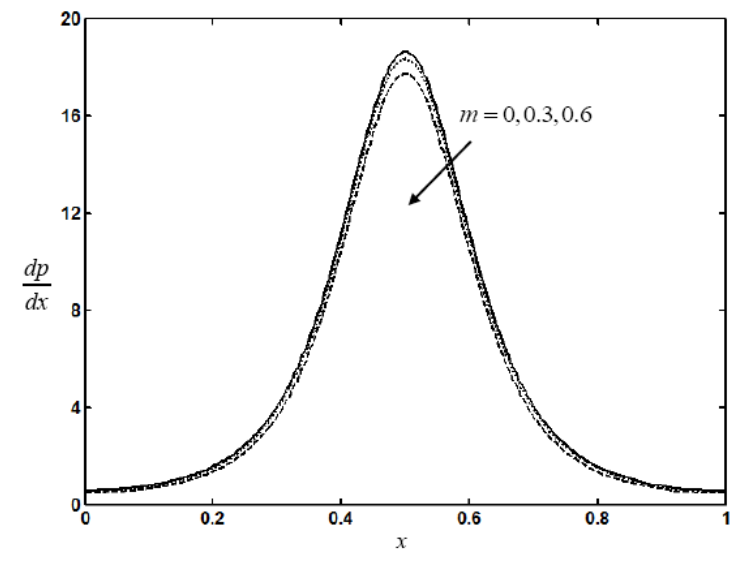

Fig. 5 The variation of the axial pressure gradient $\frac{d p}{d x}$ with $m$ for $n=0.5$,

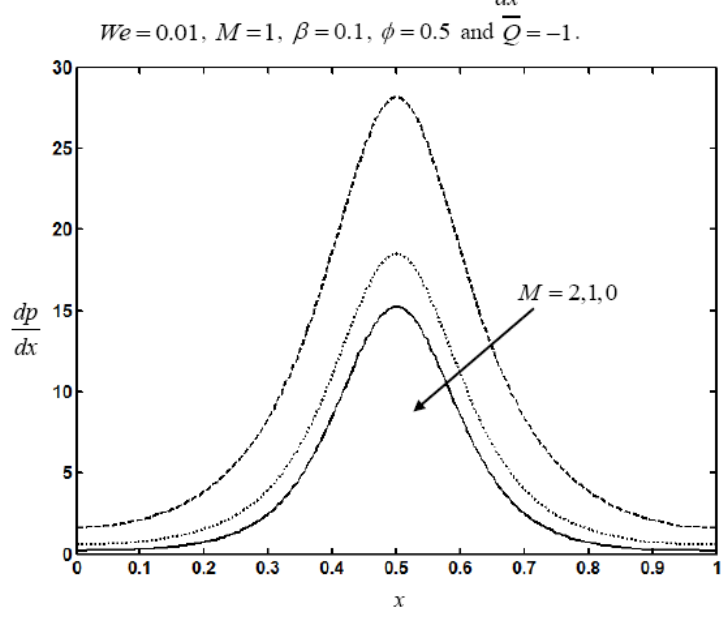

Fig. 6 The variation of the axial pressure gradient $\frac{d p}{d x}$ with $M$ for $n=0.5$, $m=0.2, W e=0.01, \beta=0.1, \phi=0.5$ and $\bar{Q}=-1$.

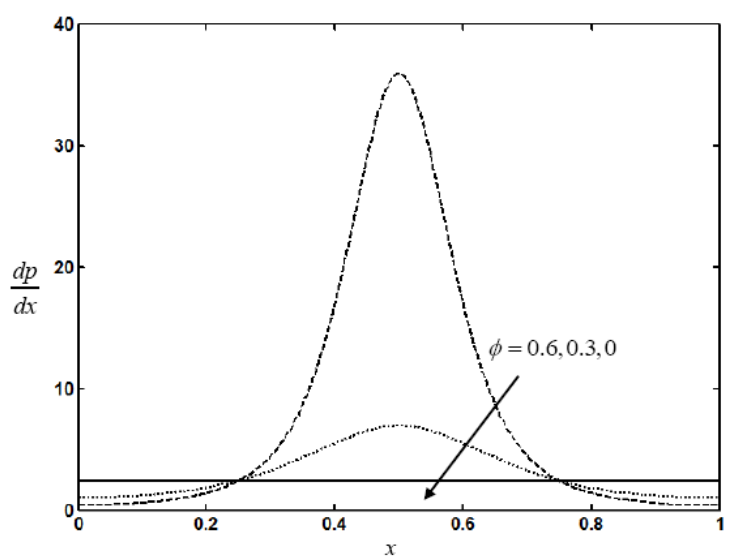

Fig. 7 The variation of the axial pressure gradient $\frac{d p}{d x}$ with $\phi$ for $n=0.5$, $m=0.2, M=1, \beta=0.1, W e=0.01$ and $\bar{Q}=-1$.
$\Delta p$

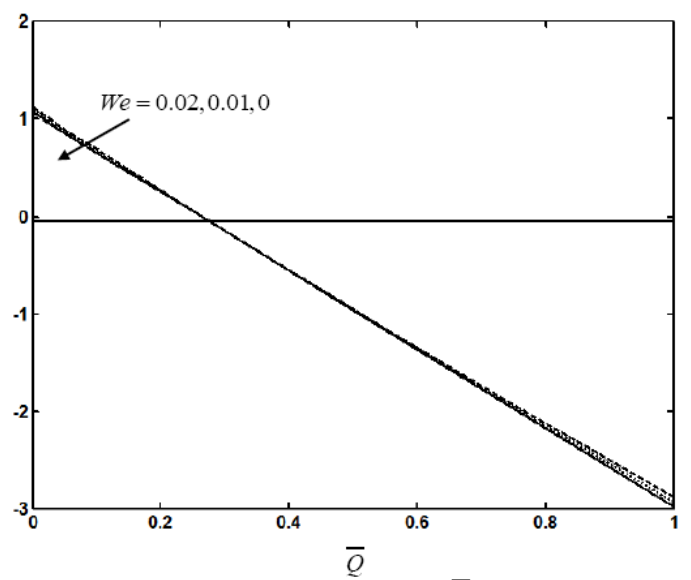

Fig. 8 The variation of the pressure rise $\Delta p$ with $\bar{Q}$ for different values of $W e$ with $n=0.5, \beta=0.1, m=0.2, M=1$ and $\phi=0.5$.

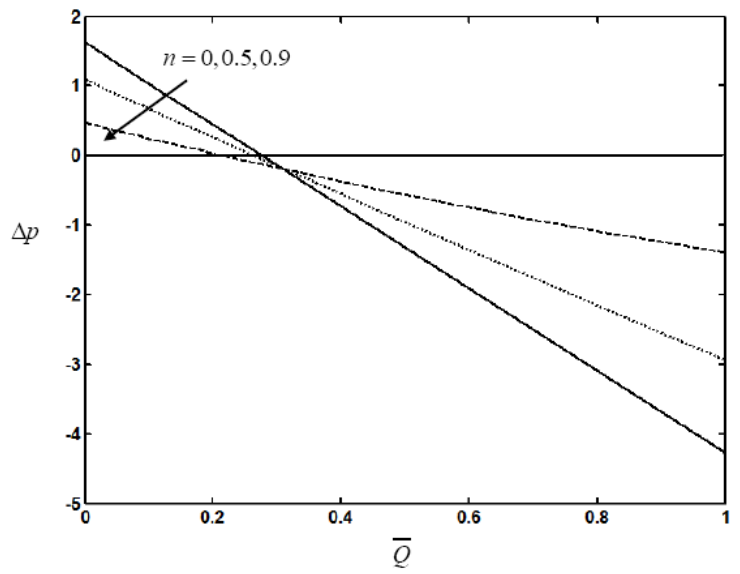

Fig. 9 The variation of the pressure rise $\Delta p$ with $\bar{Q}$ for different values of $n$ with $W e=0.01, \beta=0.1, m=0.2, M=1$ and $\phi=0.5$.

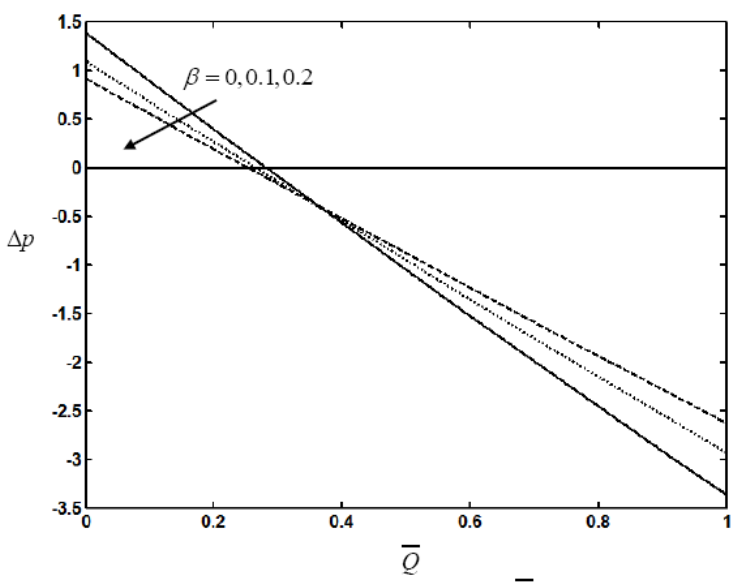

Fig. 10 The variation of the pressure rise $\Delta p$ with $\bar{Q}$ for different values of $\beta$ with $n=0.5, m=0.2, W e=0.01, M=1$ and $\phi=0.5$. 


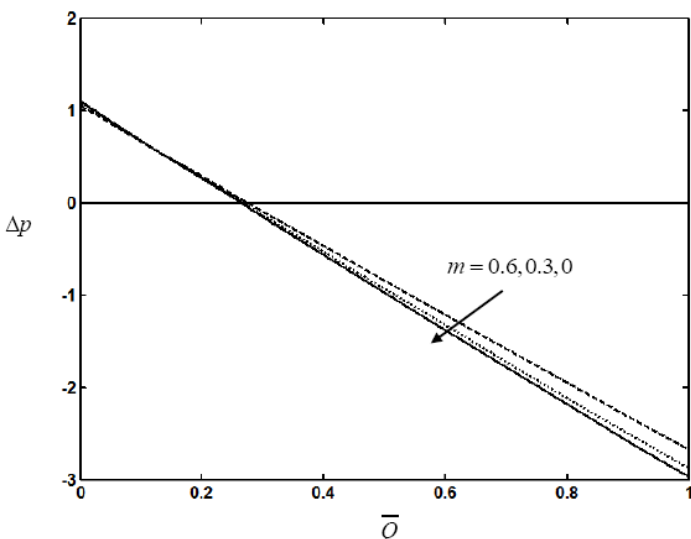

Fig. 11 The variation of the pressure rise $\Delta p$ with $\bar{Q}$ for different values of $m$ with $n=0.5, \beta=0.1, W e=0.01, M=1$ and $\phi=0.5$.

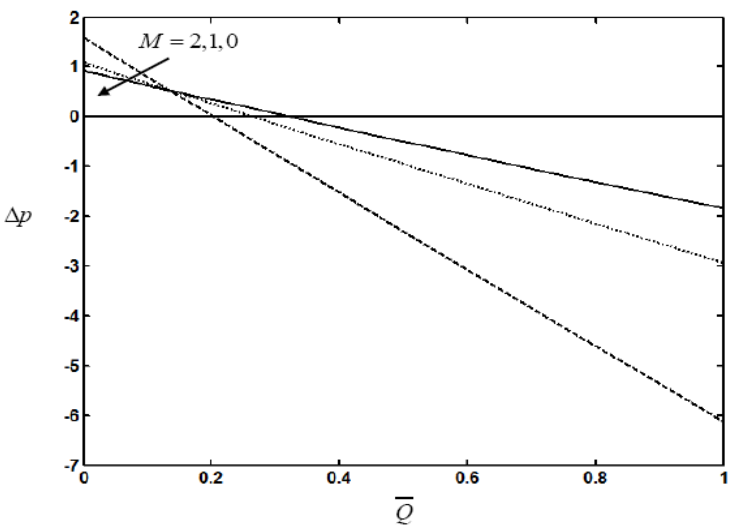

Fig. 12 The variation of the pressure rise $\Delta p$ with $\bar{Q}$ for different values of $M$ with $n=0.5, \beta=0.1, m=0.2$, We $=0.01$ and $\phi=0.5$.

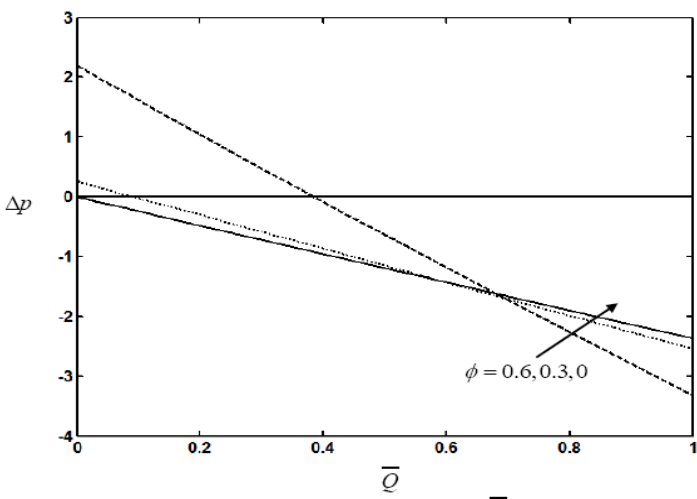

Fig. 13 The variation of the pressure rise $\Delta p$ with $\bar{Q}$ for different values of $\phi$ with $n=0.5, \beta=0.1, m=0.2, M=1$ and $W e=0.01$.

\section{CONCLUSIONS}

In this paper, we investigated the effects of slip and Hall on the peristaltic transport of a hyperbolic tangent fluid in a planar channel under the assumption of long wavelength. The expressions for the velocity and axial pressure gradient are obtained by employing perturbation technique. It is found that, the axial pressure gradient and time-averaged flow rate in the pumping region increases with increasing the Weissenberg number $W e$, the Hartmann number $M$ and the amplitude ratio $\phi$, while they decreases with increasing power-law index $n$, slip parameter $\beta$ and Hall parameter $m$.

\section{REFERENCES}

[1] Abo-Eldahab, E.M., Barakat, E.I and Nowar, K.I. Effects of Hall and ion-slip currents on peristaltic transport of a couple stress fluid, International Journal of Applied Mathematics and Physics, 2 (2) (2010), 145-157.

[2] Ai, L. and Vafai, K. An investigation of Stokes' second problem for non-Newtonian fluids, Numerical Heat Transfer, Part A, 47(2005), 955-980.

[3] Akbar, N.S., Hayat, T., Nadeem, S. and Obaidat, S. Peristaltic flow of a Tangent hyperbolic fluid in an inclined asymmetric channel with slip and heat transfer, Progress in Computational Fluid Dynamics, an International Journal, 12(5) (2012), 363-374.

[4] Ali, N., Wang, Y., Hayat, T. and Oberlack, M. Slip effects on the peristaltic flow of a third grade fluid in a circular cylindrical tube, $\mathrm{J}$. Appl. Mech., 76(2009), 1-10.

[5] Bhatti, M. M., Ali Abbas, M. and Rashidi, M. M. Effect of hall and ion slip on peristaltic blood flow of Eyring Powell fluid in a nonuniform porous channel, World Journal of Modelling and Simulation, 12(4) (2016), 268-279.

[6] Chaube, M.K., Pandey, S.K. and Tripathi, D. Slip Effect on Peristaltic Transport of Micropolar Fluid, Applied Mathematical Sciences, 4(2010), $2105-2117$.

[7] Eldabe, N.T.M., Ahmed Y. Ghaly, A.Y., Sallam, S.N., Elagamy, K and Younis, Y.M. Hall effect on peristaltic flow of third order fluid in a porous medium with heat and mass transfer, Journal of Applied Mathematics and Physics, 3(2015), 1138-1150.

[8] Elshahed, M. and Haroun, M. H. Peristaltic transport of JohnsonSegalman fluid under effect of a magnetic field, Math. Probl. Engng. 6 (2005), 663-677.

[9] El-Shehawey, E.F., El-Dabe, N.T. and El-Desoky, I. M. Slip effects on the Peristaltic flow of a Non-Newtonian Maxwellian Fluid, Acta Mech., 186(2006), 141-159.

[10] Hayat, T $\mathrm{F} \backslash$ and Ali, N. Peristaltically induced motion of a MHD third grade fluid in a deformable tube, Physica A: Statistical Mechanics and its Applications, 370(2006), 225-239.

[11] Hayat, T., Ali, N, and Asghar, S. Hall effects on peristaltic flow of a Maxwell fluid in a porous medium, Phys. Letters A, 363(2007), 397 403.

[12] Jyothi, B., Satyanarayana, B. and Subba Reddy, M.V. Slip effects on peristaltic transport of a Prandtl fluid in a channel under the effect of magnetic field, South Asian Journal of Mathematics, 5(1)(2015), 1 12 .

[13] Kwang - Hua Chu, W. and Fang, J. Peristaltic transport in a slip flow, Eur. Phys., J. B., 16(2000), 543-547.

[14] Li, A.A., Nesterov, N.I, Malikova, S.N. and Kilatkin, V.A. The use of an impulse magnetic field in the combined of patients with store fragments in the upper urinary tract. Vopr kurortol Fizide. Lech Fiz Kult, 3(1994), 22-24.

[15] Nadeem, S. and Akram, S. Peristaltic transport of a hyperbolic tangent fluid model in an asymmetric channel, Z. Naturforsch., 64a (2009), $559-567$.

[16] Nadeem, S. and Akram, S. Magnetohydrodynamic peristaltic flow of a hyperbolic tangent fluid in a vertical asymmetric channel with heat transfer, Acta Mech. Sin., 27(2) (2011), 237-250.

[17] Nadeem, S. and Akbar, S. Numerical analysis of peristaltic transport of a Tangent hyperbolic fluid in an endoscope, Journal of Aerospace Engineering, 24(3) (2011), 309-317.

[18] Noreen, S., Hayat, T. and Alsaedi, A. Study of slip and induced magnetic field on the peristaltic flow of pseudoplastic fluid, International Journal of Physical Sciences, 6(36)(2011), 8018-8026.

[19] Prasanth Reddy, D. and Subba Reddy, M.V. Peristaltic pumping of third grade fluid in an asymmetric channel under the effect of magnetic fluid, Advances in Applied Science Research, 3(6)(2012), $3868-3877$.

[20] Shapiro, A.H., Jaffrin, M.Y and Weinberg, S.L. Peristaltic pumping with long wavelengths at low Reynolds number, J. Fluid Mech. 37(1969), 799-825.

[21] Subba Narasimhudu, K. and Subba Reddy, M. V. Hall effects on the peristaltic pumping of a hyperbolic tangent fluid in a planar channel, Int. J. Mathematical Archive, 8(3) (2017), 70 - 85

[22] Subba Reddy, M.V., Jayarami Reddy, B., Nagendra, N. and Swaroopa, B. Slip effects on the peristaltic motion of a Jeffrey fluid through a porous medium in an asymmetric channel under the effect magnetic field, Journal of Applied Mathematics and Fluid Mechanics, 4(2012), 59-72 